\title{
A comprehensive study on the behavior of a rigid block on an oscillating ground with friction, elastic and viscous forces
}

\author{
M. Nicolas ${ }^{\mathrm{a}}$ \\ ${ }^{a}$ Aix-Marseille Université ; CNRS ; IUSTI, UMR 7343 ; 5 rue Enrico Fermi, 13453 \\ Marseille cedex 13, France
}

\begin{abstract}
This paper investigates the behavior of a non-linear mechanical model where a block is driven by an oscillating ground through Coulomb friction, a linear viscous damper and a linear spring. The governing equation is solved analytically for different partial configurations: friction only, friction with viscous damping, friction with a linear restoring force, and for the complete model. Using dimensionless groups, the analysis of the block motion provides a comprehensive set of information on the motion regime (stick, stick-slip or permanent sliding), on the dominant energies or forces, on the resonance and on the amplification of the ground oscillation by the system. The limit between the stick-slip regime and the permanent slipping regime is found either analytically or numerically. It is also shown that there exists a set of parameters for which the friction force, the viscous dissipative force and the elastic restoring force are equal.
\end{abstract}

Keywords: friction, oscillator, seismic response 


\section{Introduction}

While the mass-spring-dashpot system is extensively presented in physics textbooks, the addition of a strongly non linear force such as the Coulomb friction force leads to a more complex problem. Indeed, when an external energy is brought to the system not only by the spring but also through the dry friction between two solid surfaces, the friction force is both a damping force and a driving force.

The coupling of friction and an external vibration is interesting in many physical and mechanical systems. A vertical pile of rigid blocks is a paradigm for many old built structures such as greek columns [1], medieval churches [2] or five-stories wooden pagodas in Japan [3]. When the ground is shaken because of seismic activity, the friction force between the blocks may not be sufficient to prevent the collapse of the structure. Much more recently in the building history, the development of anti seismic buildings $[4,5]$ is reached by the use of soft and dissipative links between beams and columns, as sketched in Fig.1(a): a reinforced elastomer supplies elasticity while a dashpot supplies viscous dissipation between the pillar and the beam. Another recent system for seismic-response reduction is the friction pendulum system (FPS) where a contact element coated with teflon slides on a spherical concave surface (as sketched on Fig .1b). A simple non-linear mechanical model of the horizontal restoring force implies a Coulomb friction force as well as an elastic restoring force [6]. Despite these solutions are already implemented on actual buildings, the understanding of the relative importance of the different mechanical elements is still to establish.

This paper aims to bring a fundamental insight on the energy transfer 


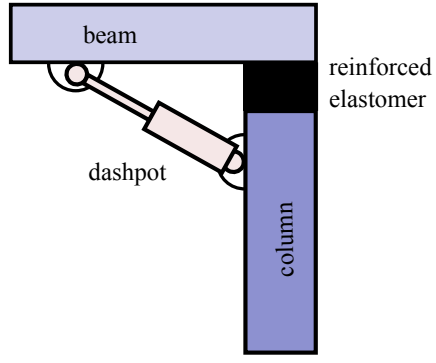

(a)

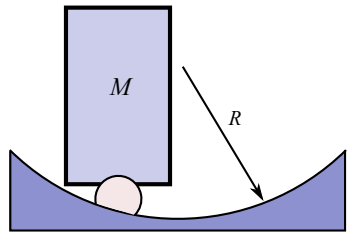

(b)

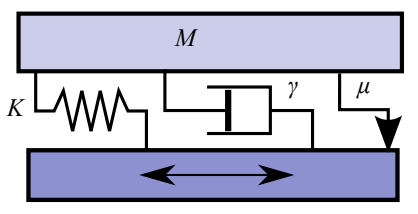

(c)

Figure 1: (a) Sketch of a damping link between a column and a beam in a anti seismic building. (b) Sketch of a simple friction pendulum system. (c) Corresponding mechanical model studied in this paper.

through a friction-elastic-viscous link between an oscillating ground and a rigid block. We focus here on the role of the non-linear friction force on the resonance frequency and on the relative motion between the block and the ground. The transition between the stick regime, the stick-slip regime and the permanent slip regime is investigated both theoretically and numerically with the use of dimensionless groups.

\section{The governing equation and analysis method}

\subsection{The model}

The mechanical model of the pillar-beam link is presented on Fig. 1(c). We consider a single block of mass $M$ linked to a horizontally shaking ground through a friction-viscous-elastic set of forces. We note $X$ the position of the block relatively to the ground, $\dot{X}$ its velocity, $K$ the spring stiffness (in $\mathrm{N} \cdot \mathrm{m}^{-1}$ ), $\gamma$ the viscous dissipation constant (in $\mathrm{kg} \cdot \mathrm{s}^{-1}$ ), and $\mu(\dot{X})$ the friction law. The ground has a monochromatic horizontal motion with an amplitude 


\begin{tabular}{|c|c|c|}
\hline & static friction coefficient & kinematic friction coefficient \\
\hline friction model 1 & $\mu_{s}$ & $\mu_{k}=\mu_{s}$ \\
\hline friction model 2 & $\mu_{s}$ & $\mu_{k}=0.7 \mu_{s}$ \\
\hline
\end{tabular}

Table 1: Description of the two friction models used in this study.

$A_{0}$, a frequency $\Omega / 2 \pi$, and an initial phase $\varphi$. This system is slightly different than the model studied by Hundal [7], López [8] or Chatterjee [9]: in this present model, the spring and dashpot is a link between the mass and the oscillating ground and not between the mass and a non-moving wall. Taking the oscillating ground as a reference frame, the relative motion equation of the block is

$$
M \ddot{X}=-\mu(\dot{X}) M g \frac{\dot{X}}{|\dot{X}|}-K X-\gamma \dot{X}-M A_{0} \Omega^{2} \cos (\Omega T+\varphi) .
$$

In this study, two Coulomb friction models $\mu(\dot{X})$ are considered. The first and simplest model is the Coulomb friction model with a unique friction coefficient $\mu_{s}$. The second model introduces a kinematic friction coefficient $\mu_{k}$ when the block slides on the ground, usually lower than the static friction coefficient $\mu_{s}$. The table 1 summarizes the values for models 1 and 2 . Many other friction models exist (for example see the review [10]) but since they need more than two parameters, they we prefer simple Coulomb models to highlight the role of the friction force.

Introducing the following dimensionless variables and groups $x=\frac{X}{A}, \quad \dot{x}=\frac{\dot{X}}{\sqrt{A_{0} g}}, \quad k=\frac{K}{M} \frac{A_{0}}{g}, \quad \eta=\frac{\gamma}{M} \sqrt{\frac{A_{0}}{g}}, \quad \alpha=\frac{A_{0} \Omega^{2}}{\mu_{s} g}, \quad \omega=\sqrt{\alpha \mu_{s}}$ 
the motion equation (1) becomes

$$
\ddot{x}+\mu(\dot{x}) \frac{\dot{x}}{|\dot{x}|}+k x+\eta \dot{x}+\omega^{2} \cos (\omega t+\varphi)=0 .
$$

In this non-dimensional model, the governing parameter is the reduced acceleration $\alpha$, and the non-dimensional frequency is $\omega=\sqrt{\alpha \mu_{s}}$, which depends on the static friction coefficient. In addition, we assume the following initial conditions : $x(0)=0, \dot{x}(0)=0$ and we set $\varphi=\pi / 2$ to ensure an initial zero acceleration of the ground.

\subsection{Analysis method and motion characteristics}

Despite the non-linear behavior of the friction force, the motion equation (2) is piecewise linear, and the block either sticks or slides on the ground. With the given initial conditions (see above), the block starts to slide at a time $t_{0}>0$ when the inertia force overcomes the static friction force. An analytical solution of Eq. (2) may be found, and since two integration constants arise, these constants are solved using the initial conditions $x\left(t_{0}\right)$ and $\dot{x}\left(t_{0}\right)=0$. The block then stops at a time $t_{1}>t_{0}$ when its velocity is zero again $\dot{x}\left(t_{1}\right)=0$. This time $t_{1}$ is numerically solved using a Newton method. At $t=t_{1}$ the block either sticks to the ground if the sum of the inertia and elastic forces is lower than the static friction force, or slides again with a velocity sign change if the sum of the inertia and elastic forces is greater than the static friction force. The first case leads to a stick-slip motion and the block sticks until time $t_{2}>t_{1}$ where the start condition is reached again i.e. when the sum of the inertia force and the elastic force is greater than the static friction force. The second case leads to a permanent slipping motion and the next start time is simply $t_{2}=t_{1}$, with $t_{2}-t_{1}=\pi / \omega$, the half-period 
of oscillation of the ground. The sequel of the motion is calculated with the same method as previously with a new start time $t_{2}$ instead of $t_{0}$ for the computation of the integration constants, and subsequently the analytical solution of Eq. (2) is given through a list of $\left\{t_{i}\right\}$ times, where even $i$ are start indexes, and odd $i$ are stop indexes. An illustration of the $t_{i}$ times is given on Fig. 2(a) for a stick-slip motion. We checked our analytical method with a numerical solving of Eq. (2) using an implicite numerical scheme with a10 ${ }^{-3}$ time-step. Both methods were implemented using Python packages and codes.

The block behavior is characterized with different measurable quantities. First, we measure the the block amplitude $A$ which is half of the peak-topeak block displacement $x$. We also introduce the time-ratio $\zeta$ which is the time when the block slips on the ground compared to the total time:

$$
\zeta=\frac{\Delta t_{\text {slip }}}{\Delta t_{\text {slip }}+\Delta t_{\text {stick }}}=\frac{\left(t_{i+3}-t_{i+2}\right)+\left(t_{i+1}-t_{i}\right)}{t_{i+4}-t_{i}}
$$

where $i$ is the index of a start time. A permanent stick regime is thus observed for $\zeta=0$ (ST), a stick and slip motion for $0<\zeta<1$ (SS) and a permanent slip regime is observed for $\zeta=1(\mathrm{SL})$. A last relevant quantity is the root mean squared velocity defined by

$$
\dot{x}_{r m s}=\sqrt{\frac{1}{\tau} \int_{t}^{t+\tau} \dot{x}^{2}\left(t^{\prime}\right) d t^{\prime}}
$$

\subsection{Forces and energies}

The relative importance of the three forces in the system can be assessed through non dimensional groups. Assuming that a steady state is established with a bounded oscillation amplitude, a generic solution is $x(t)=$ 
$A\left(\mu_{s}, \mu_{k}, k, \eta\right) F(\omega t)$ where $F$ is a periodic bounded function with $|\max (F)|=$ 1. We therefore introduce

$$
\rho_{e v}=\frac{k^{2}}{\eta^{2} \alpha \mu_{s}}, \quad \rho_{v f}=\frac{\eta^{2} A^{2} \alpha \mu_{s}}{\mu_{k}^{2}}, \quad \rho_{e f}=\frac{k A}{\mu_{k}}
$$

which are the ratio of the elastic force to the viscous force, the ratio of the viscous force to the friction force, and the ratio of the elastic force to the friction force respectively.

By multiplying Eq. (2) with the relative velocity $\dot{x}$ and integrating over time, the energy conservation equation is

$$
\int_{0}^{t} \ddot{x} \dot{x} d t^{\prime}+\int_{0}^{t} \dot{x} x d t^{\prime}+\mu_{k} \int_{0}^{t}|\dot{x}| d t^{\prime}+\eta \int_{0}^{t} \dot{x}^{2} d t^{\prime}+\alpha \mu_{s} \int_{0}^{t} \dot{x} \cos \left(\omega t^{\prime}+\varphi\right) d t^{\prime}=0
$$

which may be written as

$$
e_{k}+e_{p}+e_{f}+e_{v}=-w_{i}
$$

where $e_{c}$ is the kinetic energy, $e_{p}$ is the potential energy, $e_{f}$ is the friction dissipated energy, $e_{v}$ is the viscous dissipation energy, and $w_{i}$ is the work of the inertia force.

If the time integration is taken over one cycle (between $t$ and $t+\tau$, $\tau=2 \pi / \omega)$, there is no variation of the kinetic and potential energies because of the periodicity of the $x(t)$ and $\dot{x}(t)$ functions, and the energy conservation equation is reduced to

$$
\Delta e_{f}+\Delta e_{v}+\Delta w_{i}=0
$$

The first term $\Delta e_{f}$ is the energy dissipated by Coulomb friction during sliding. Over one cycle of oscillation, it is equivalent to

$$
\Delta e_{f}=\mu_{k} \int_{t}^{t+\tau}\left|\dot{x}\left(t^{\prime}\right)\right| d t^{\prime}=\mu_{k} \int_{t}^{t+\tau}|d x|=4 \mu_{k} A
$$




\begin{tabular}{|c|c|c|c|c|}
\hline configuration & friction & viscous damping & elasticity & damping \\
\hline configuration 1 & $\bullet$ & & & \\
\hline configuration 2 & $\bullet$ & $\bullet$ & & \\
\hline configuration 3 & $\bullet$ & & $\bullet$ & \\
\hline configuration 4a & $\bullet$ & $\bullet$ & $\bullet$ & weak \\
\hline configuration 4b & $\bullet$ & $\bullet$ & $\bullet$ & critical \\
\hline configuration 4c & $\bullet$ & $\bullet$ & $\bullet$ & strong \\
\hline
\end{tabular}

Table 2: Configurations studied in this paper.

where $A$ is the amplitude of the block motion. The second term is the energy dissipated by viscous dissipation over one cycle:

$$
\Delta e_{v}=\eta \int_{t}^{t+\tau} \dot{x}^{2}\left(t^{\prime}\right) d t^{\prime}=\eta \tau\left(\dot{x}_{r m s}\right)^{2} .
$$

Finally, the the third term of Eq. (8) is the work of the inertia force

$$
\Delta w_{i}=\omega^{2} \int_{t}^{t+\tau} \dot{x}\left(t^{\prime}\right) \cos \left(\omega t^{\prime}+\varphi\right) d t^{\prime}
$$

which is the energy input per cycle of ground oscillation.

In this paper, we investigate separately the role of the friction force, of the elastic restoring force and of the viscous dissipation force, and we seek to highlight the effects of these forces on the amplitude $A$, and on the time-ratio $\zeta$ depicting the sliding regimes. The different configurations are summarized in table 2. All the results presented in this paper correspond to a permanent periodic motion, i.e. when the transient motion has vanished. 


\section{Results for configurations $1-3$}

In this section we study three partial models where only the friction force applies (configuration 1), the friction force and the viscous dissipative force are present (configuration 2) and where the friction force is coupled with the elastic restoring force (configuration 3).

\subsection{Configuration 1: friction only}

When the friction is the only applied force $(k=0, \eta=0)$, it plays the ambiguous role of the driving force and the damping force. The Eq. (2) then reduces to

$$
\ddot{x}=-\mu(\dot{x}) \frac{\dot{x}}{|\dot{x}|}-\omega^{2} \cos (\omega t+\varphi) .
$$

Such a mechanical model is known to present two acceleration thresholds. The non-dimensional acceleration threshold between the sticking regime and the stick-slip regime is simply $\alpha_{S S}=1$. The criterion $\alpha \geqslant \alpha_{S L}$ for the permanent slipping regime may be calculated as follows. Naming

$$
t_{0}=\omega^{-1}\left[\arccos \left(-\mu_{s} / \omega^{2}\right)-\varphi\right]
$$

the time when the block starts to slide and $t_{f}$ the time when the block stops sliding, the sliding duration $t_{f}-t_{0}$ is governed by

$$
\mu_{k}\left(t_{f}-t_{0}\right)+\omega\left[\sin \left(\omega t_{f}+\varphi\right)-\sin \left(\omega t_{0}+\varphi\right)\right]=0 .
$$

Since the block is sliding at any time when $t_{f}-t_{0}=\pi / \omega$ (half of a period), this gives a criteria on the vibration parameter

$$
\alpha_{S L}=\sqrt{1+\frac{\pi^{2}}{4}\left(\frac{\mu_{k}}{\mu_{s}}\right)^{2}} .
$$


With a unique friction coefficient $\left(\mu_{s}=\mu_{k}\right)$, this model has been proposed as early as the 1930's by Den Haartog [11], then by Alspaugh [12], and extended by Westermo \& Udwadia [13] with $\alpha_{S L}=\sqrt{1+\pi^{2} / 4} \approx 1.86$ (see López [8] or Benedetti et al. [14] for the analytical calculation of $\alpha_{S L}$ ).

During the sliding, the solution of Eq. (12) is

$$
x(t)=c_{1}+c_{2} t-\frac{1}{2} \mu_{k} t^{2}+\cos (\omega t+\varphi)=0
$$

where the constants $c_{1}$ and $c_{2}$ are the solutions of

$$
\left(\begin{array}{cc}
1 & t_{0} \\
0 & 1
\end{array}\right)\left(\begin{array}{c}
c_{1} \\
c_{2}
\end{array}\right)=\left(\begin{array}{c}
x_{0}+\frac{1}{2} \mu_{k} t_{0}^{2}-\cos \left(\omega t_{0}+\varphi\right) \\
\dot{x}_{0}+\mu_{k} t_{0}+\omega \sin \left(\omega t_{0}+\varphi\right)
\end{array}\right)
$$

An example of the block trajectory and its corresponding velocity is shown on Fig. 2a, showing a typical stick and slip motion for $\alpha=1.2$. It must be noted that even with a single friction coefficient (red curves), the stick-slip regime exists. This model is indeed different from the traditional masson-belt model where the stick-slip phenomenon occurs only for a kinetic friction coefficient different (and lower) than $\mu_{s}$. With the friction model 2 (black curves), the block shows a larger displacement amplitude and a larger peak velocity (Fig. 2b) due to the lower friction coefficient during the sliding motion. We show the influence of $\alpha$ on $\zeta$ and $A$ on Fig. 2(e) and (f) respectively. With this friction-only model, the block amplitude $A$ increases with the vibration parameter $\alpha$ from the unity threshold and tends towards unity for a large acceleration parameter $\alpha$ (Fig. 2f), whatever the friction model. There is no obvious discontinuity on the amplitude curves when the motion regime changes from stick-slip (SS) to permanent slip (SL). For a 

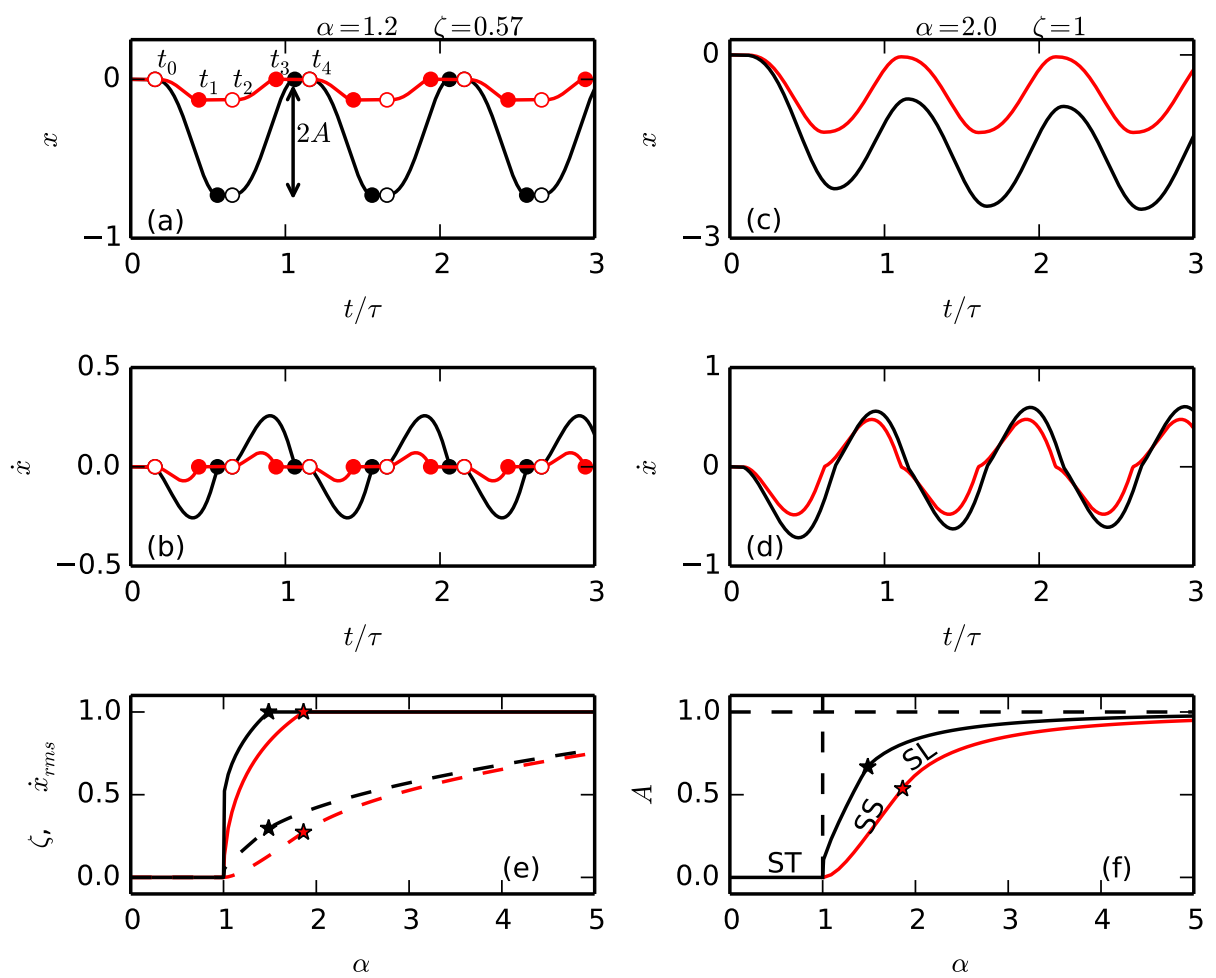

Figure 2: Configuration 1. (a) position and (b) velocity for $\alpha=1.2$, (c) position and (d) velocity for $\alpha=2.0$. The red curves are computed for the friction model 1 , and the black curves are computed for the friction model 2. Full symbols indicates a stop of the motion whereas empty symbols indicates a start of the motion. For this example the static friction coefficient is $\mu_{s}=0.25$. (e) solid line: sliding time-ratio $\zeta$ increasing with the acceleration parameter $\alpha$, for friction model 1 (red curve) and model 2 (black curve). Dashed line: characteristic velocity $\dot{x}_{r m s}$. (f) the block amplitude $A$ varying with the $\alpha$ vibration parameter for the friction model 1 (red curve) and the friction model 2 (black curve). The stars indicates the threshold between the stick-slip regime and the permanent slip regime. 
larger acceleration $\alpha>\alpha_{S L}$, the block slides continuously (Fig. 2c,d), the direction change is indicated by a slope discontinuity on the velocity curve.

\subsection{Configuration 2: friction and viscous dissipation}

When the viscous dissipation term is non-zero, without the elastic restoring force $(k=0)$, the governing equation is

$$
\ddot{x}+\mu(\dot{x}) \frac{\dot{x}}{|\dot{x}|}+\eta \dot{x}+\omega^{2} \cos (\omega t+\varphi)=0 .
$$

with an explicit solution for $t \geqslant t_{0}$ (when sliding)

$x(t)=c_{1}-\frac{c_{2}}{\eta} \mathrm{e}^{-\eta t}-\frac{\mu_{k}}{\eta \omega}(\omega t+\varphi)+\frac{1}{\eta^{2}+\omega^{2}}\left[\omega^{2} \cos (\omega t+\varphi)-\eta \omega \sin (\omega t+\varphi)\right]$

where the constants are the solutions of

$$
\begin{aligned}
& \left(\begin{array}{cc}
1 & -\frac{1}{\eta} \mathrm{e}^{-\eta t_{0}} \\
0 & \mathrm{e}^{-\eta t_{0}}
\end{array}\right)\left(\begin{array}{c}
c_{1} \\
c_{2}
\end{array}\right) \\
= & \left(\begin{array}{c}
x_{0}+\frac{\mu_{k}}{\eta \omega}\left(\omega t_{0}+\varphi\right)-\frac{1}{\eta^{2}+\omega^{2}}\left[\omega^{2} \cos \left(\omega t_{0}+\varphi\right)-\eta \omega \sin \left(\omega t_{0}+\varphi\right)\right] \\
\dot{x}_{0}+\frac{\mu_{k}}{\eta}+\frac{\omega^{2}}{\eta^{2}+\omega^{2}}\left[\omega \sin \left(\omega t_{0}+\varphi\right)+\eta \cos \left(\omega t_{0}+\varphi\right)\right]
\end{array}\right)
\end{aligned}
$$

We show on Figs. 3(a,b) the $\alpha_{S L}(\eta)$ curves for both friction models. For a weak dissipation parameter $(\eta \leqslant 2)$, these curves may be well approximated by

$$
\alpha_{S L}(\eta)=\left[1+\left(-0.48 \frac{\mu_{s}}{\mu_{k}}+1.18\right) \eta\right] \sqrt{1+\frac{\pi^{2}}{4}\left(\frac{\mu_{k}}{\mu_{s}}\right)^{2}}
$$

with a linear increase of the threshold with the viscous parameter $\eta$. This expression is plotted as blue dotted lines in Figs. 3(a,b).

Compared to the configuration 1, adding a viscous dissipative force helps to reduce the block amplitude, as shown on Fig. 3(c), computed for $\alpha=2.5$. 

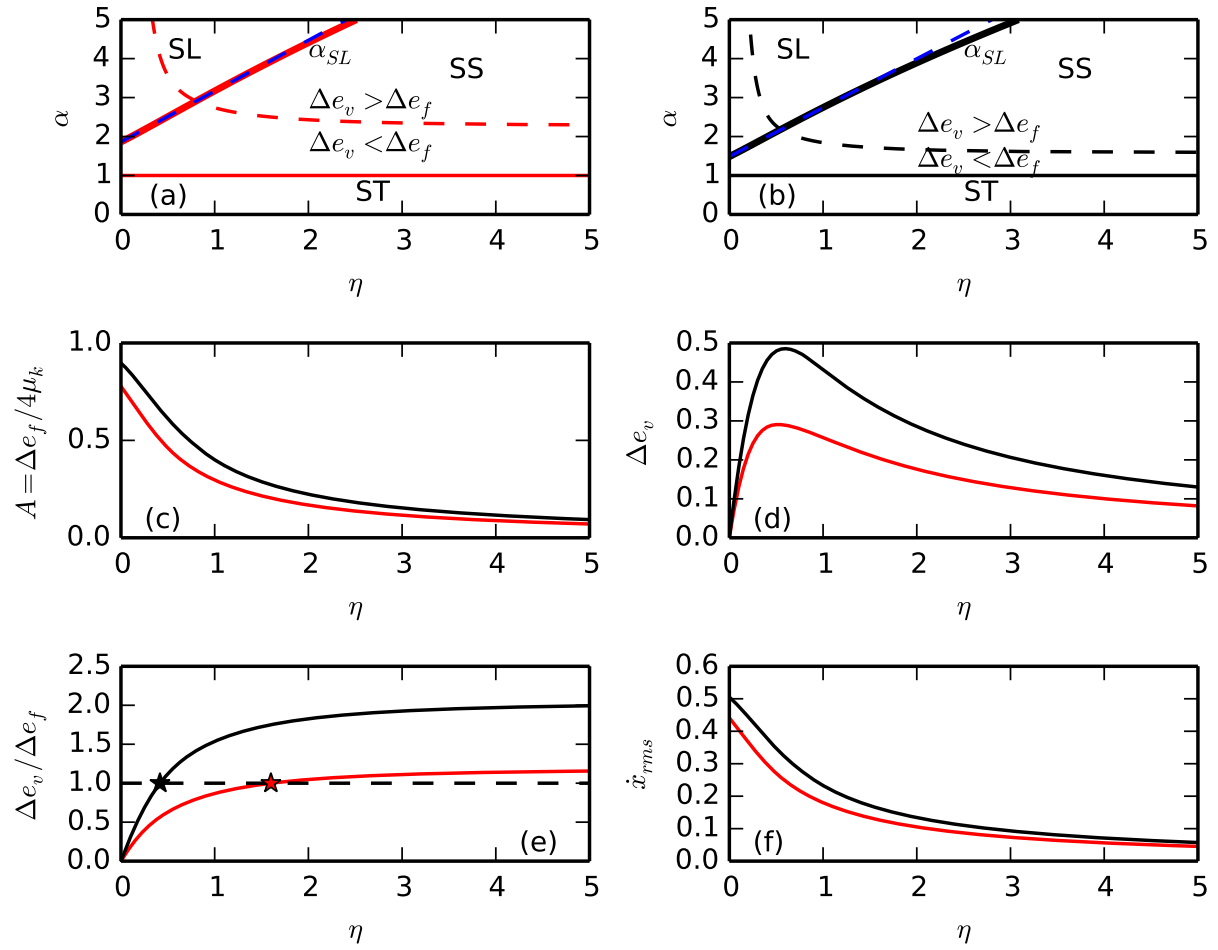

Figure 3: Configuration 2. (a) the permanent slip threshold $\alpha_{S L}$ (bold red line) and the $\rho_{v f}=1$ line (dashed red line) as a function of the viscous parameter $\eta$ for friction model 1 . (b) the permanent slip threshold $\alpha_{S L}$ (bold black line) and the $\rho_{v f}=1$ line (dashed black line) as a function of the viscous parameter $\eta$ for friction model 2. The blue dotted lines in $(\mathrm{a}, \mathrm{b})$ represent the expression (21) for both friction models. (c) Block amplitude vs $\eta$ for friction model 1 (red) and friction model 2 (black). (d) The variation of the energy dissipated by the dashpot during one vibration cycle. (e) The ratio $\Delta e_{v} / \Delta e_{f}$ as a function of $\eta$. The stars indicate $\Delta e_{v}=\Delta e_{f}$. (f) The mean rms velocity as a function of $\eta$. Plots (c)-(f) are computed with $\alpha=2.5$. 
As a consequence, the energy dissipated by friction $\Delta e_{f}$ is also decreasing with an increasing viscous parameter $\eta$. The energy dissipated by the dashpot $\Delta e_{v}$ increases for low values of $\eta$, reaches a maximum, then decreases for increasing $\eta$ (Fig 3d). And the ratio $\Delta e_{v} / \Delta e_{f}$ as a function of $\eta$ shows that there exists a $\eta^{*}(\alpha)$ value which separates two regimes of dissipation: a viscous regime for $\eta>\eta^{*}$, and a friction regime for $\eta<\eta^{*}$ (Fig 3e). This threshold value depends on $\alpha$ and the limit curve separating these two dissipation regimes is plotted as a dotted line on Figs 3(a,b). The viscous parameter $\eta$ has a strong effect on the characteristic velocity $\dot{x}_{r m s}$ (Fig. 3f), very similar to the amplitude.

\subsection{Configuration 3: friction + spring}

When an elastic restoring force is coupled with the friction force, the motion equation is

$$
\ddot{x}+\mu(\dot{x}) \frac{\dot{x}}{|\dot{x}|}+k x+\omega^{2} \cos (\omega t+\varphi)=0 .
$$

and its solution is

$$
x(t)=c_{1} \cos (\sqrt{k} t)+c_{2} \sin (\sqrt{k} t)-\frac{\mu_{k}}{k}-\frac{\omega^{2}}{k-\omega^{2}} \cos (\omega t+\varphi)
$$

where the constants $c_{1}$ and $c_{2}$ are the solutions of

$$
\begin{aligned}
\left(\begin{array}{cc}
\cos \left(\sqrt{k} t_{0}\right) & \sin \left(\sqrt{k} t_{0}\right) \\
-\sqrt{k} \sin \left(\sqrt{k} t_{0}\right) & \sqrt{k} \cos \left(\sqrt{k} t_{0}\right)
\end{array}\right)\left(\begin{array}{c}
c_{1} \\
c_{2}
\end{array}\right) \\
=\left(\begin{array}{c}
x_{0}+\frac{\mu_{k}}{k}+\frac{\omega^{2}}{k^{2}-\omega^{2}} \cos \left(\omega t_{0}+\varphi\right) \\
\dot{x}_{0}-\frac{\omega^{3}}{k^{2}-\omega^{2}} \sin \left(\omega t_{0}+\varphi\right)
\end{array}\right)
\end{aligned}
$$

In this configuration, the friction force is the only dissipation force when sliding, while the static friction force and the elastic force are driving the 
block and thus are injecting energy into the system. Since the friction dissipation force is finite, the block may experience a long transient before a steady oscillating state is reached.

Some typical behaviors are shown on figure 4 for two different accelerations, $\alpha=1.2(\mathrm{a}, \mathrm{b}, \mathrm{c})$ and $\alpha=3(\mathrm{~d}, \mathrm{e}, \mathrm{f})$, and for the friction model 1. A first obvious effect of the elastic restoring force is to put the block back to a zero mean position, even if the block is displaced at the start of the motion. While in the SS regime the transient is short and a steady-state is reached after a few cycles, the transient depends on the stiffness $k$ value in the permanent slip regime. For $k<k_{r}$, the transient is short (Fig. 4d), while for $k>k_{r}$ the transient may last more than 10 oscillation cycles (Fig. 4f). Here the threshold $k_{r}$ value is a resonant stiffness simply expressed by

$$
k_{r, S L}=\alpha \mu_{s},
$$

in the SL regime, where the oscillation amplitude increases indefinitely (Fig. $4 \mathrm{e})$.

Despite an analytical solution for this configuration, it would be cumbersome to derive an analytical expression for the permanent slip vibration parameter $\alpha_{S L}$ as a function of $k$. A numerical solution is thus proposed here. The figure $5(\mathrm{a}, \mathrm{b})$ summarize the motion regimes, showing the $\alpha_{S L}(k)$ curves for the two friction models in the $(k, \alpha)$ space. The curve separating the SS and the SL regimes has a non trivial shape. For $4.1<k<5.3$ with friction model 1 or $3.7<k<5.4$ with friction model 2 , an increase of $\alpha$ from 1 causes the system to have a SS/SL/SS/SL sequence. Moreover, for a low value of $k$, with the friction model 1 (Fig. 5a), the permanent slip parameter $\alpha_{S L}$ decreases to a minimum then increases with a nearly constant slope. With the 
friction model 2, the $\alpha_{S L}(k)$ curves also decreases but intercepts the $\alpha=1$ permanent stick boundary. This shows that for $0.18<k / \mu_{s}<0.41$ the block can not show a stick-slip motion. The limit $\rho_{e f}=1$ is drawn as a magenta curve (Fig. 5a) and shows that the elastic force is dominant except for a very low value of the elastic parameter $(k<0.2)$ or for a low acceleration. For the friction model 2, the same trend is observed for the elastic parameter (the friction force is dominant for $k<0.15$ ), whatever the acceleration parameter.

Without a strong dissipation force, the amplitude diverges at the resonance. The figures $5(\mathrm{c}, \mathrm{d})$ show the curves $A(\alpha)$ for both friction models and for two values of the stiffness : $k=0.5$ and $k=1$. These curves illustrate the non trivial coupling between the elastic and the friction forces. Without a friction force, the simple undamped and forced harmonic oscillator shows a resonance curve made of two monotonic parts separated by a resonance singularity. For this system, the friction induces sub-harmonic and superharmonic resonances, as shown by the amplitude local peaks of the $A(\alpha)$ curve.

The effect of the $k$ normalized spring stiffness on the amplitude is shown on Fig. 5(e) for $\alpha=1.2$ (solid lines) and $\alpha=3.0$ (dashed lines). Results for both friction models are presented. For $\alpha=1.2$ and with $\mu_{s}=\mu_{k}$, the system is in the SL regime, and the $A(k)$ curve exhibits a finite maximum for a pseudo-resonance $k_{r}$ value. The other curves exhibit a classical resonance phenomena, with a continuous increase of the block amplitude with time. Indeed, at the resonance the amplitude $A_{r}$ increases linearly with time following

$$
A_{r}(t)=\beta t
$$



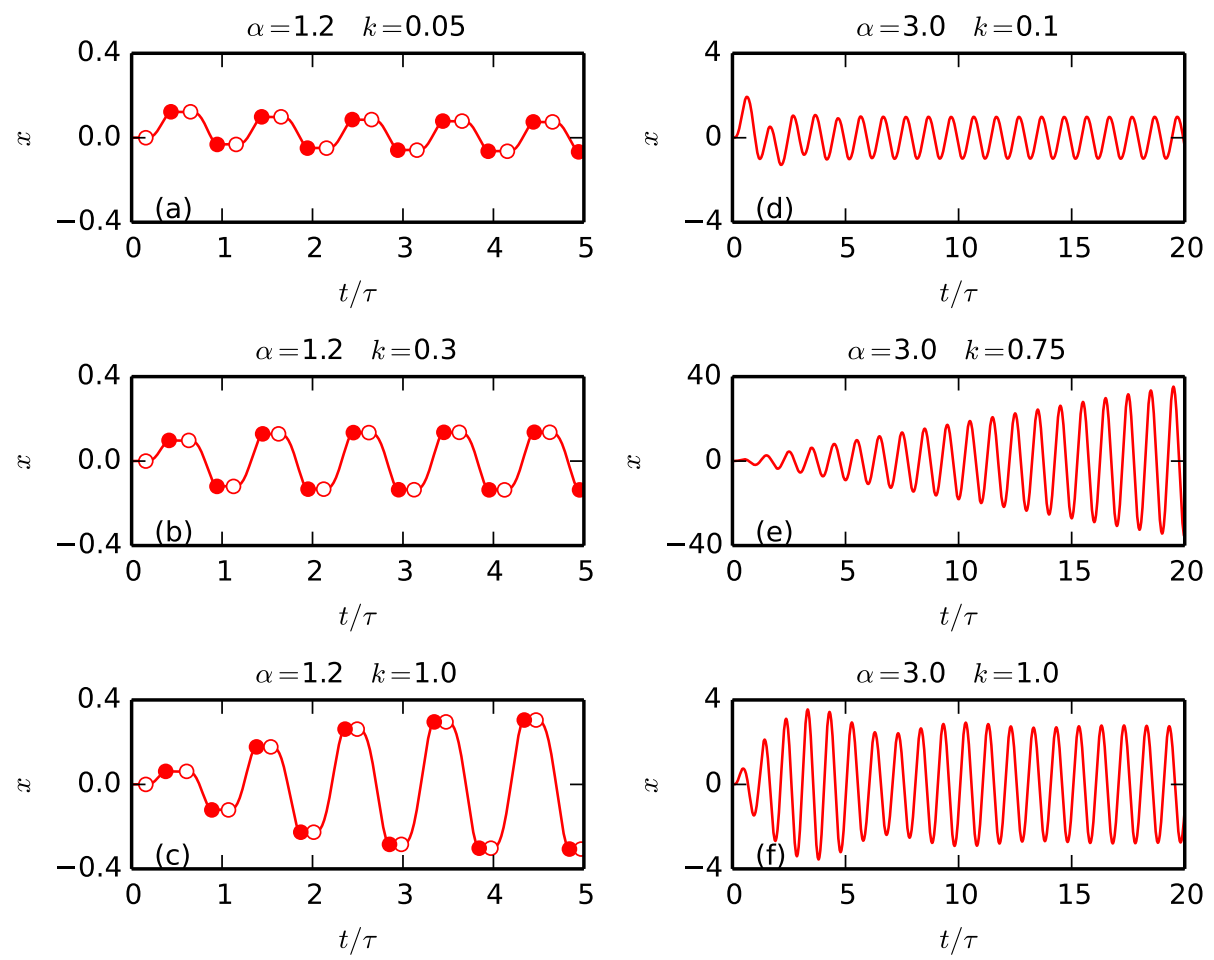

Figure 4: Configuration 3. examples of the motion of the block $x(t)$ in the stick-slip regime ( $\mathrm{a}, \mathrm{b}, \mathrm{c}: \alpha=1.2)$ and in the permanent slip regime (d,e,f: $\alpha=3)$. Curves are computed for the friction model 1 . The full symbols indicates a stop of the motion whereas empty symbols indicates a start of the motion. 

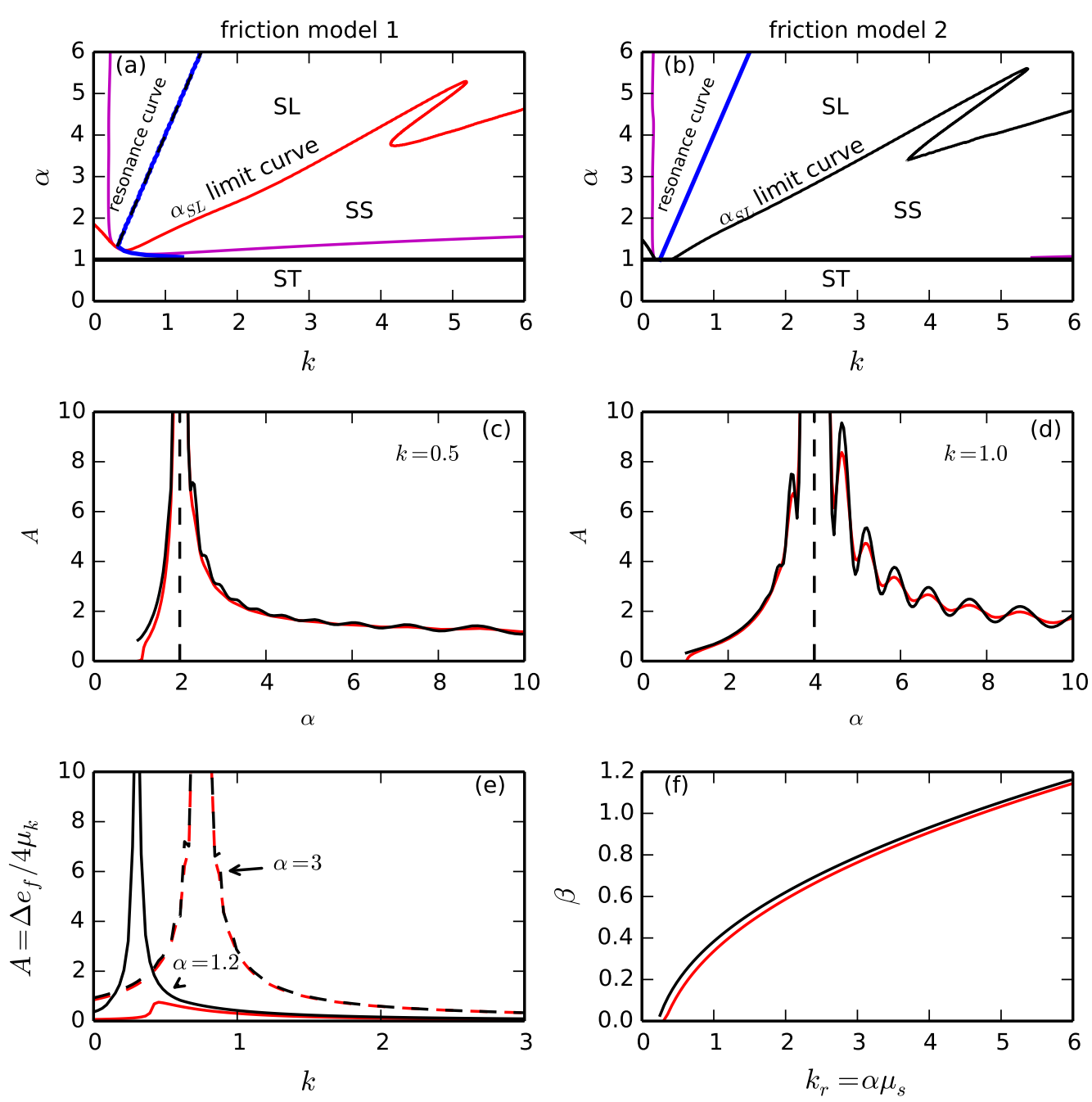

Figure 5: Configuration 3. Motion regimes in the $(k, \alpha)$ space for friction model 1 (a) and friction model 2 (b). (c) Amplitude $A(\alpha)$ for $k=0.5$ (d) Amplitude $A(\alpha)$ for $k=1$ .The blue curves indicate the resonance condition in the SL regime, and the green curve indicate the pseudo-resonance condition in the SS regime. (e) The block amplitude as a function of the stiffness $k$ for $\alpha=1.2$ (solid lines) and $\alpha=3$ (dashed lines) for friction model 1 (red) and friction model 2 (black). (f) The amplitude growth factor $\beta$ at the resonance. 
with $\beta$ as a growth coefficient depending mainly on $k$ and weakly on the friction model (Fig. 5f).

This configuration is relevant for the friction pendulum system, where the stiffness is $K_{F P S}=M g / R$ ( $M$ is here the mass of the pillar and $R$ is the curvature radius of the FPS, see Fig. 1b). In our model, the dimensionless stiffness is then $k=A_{0} / R$, the ratio of the horizontal ground displacement on the curvature radius. In an actual FPS, the curvature is of the order of one meter, and a horizontal ground displacement is typically 1 to $10 \mathrm{~cm}$ during an earthquake. This leads to a $10^{-2}$ to $10^{-1}$ value for $k$.

\section{Results for the complete model}

We now consider the solutions of Eq. (2) without neglecting any force. For a simple forced and damped oscillator, it is known that three motion behaviors exist, depending on the respective values of the damping parameter $\eta$ and the spring stiffness $k$. With our symbols, the critical damping is expressed by

$$
\eta^{2}-4 k=0
$$

and we provide in the appendix the explicit solutions of Eq. (2) for the underdamped behavior $\left(\eta^{2}-4 k<0\right)$, the critically damped oscillator $\left(\eta^{2}-\right.$ $4 k=0)$ and the overdamped behavior $\left(\eta^{2}-4 k>0\right)$.

A first result is a mapping of the block behavior in the $(k, \alpha)$ plane for $\eta=0.5$ (Fig. 6). For $\alpha \geqslant 1$, the relative motion is non zero and the boundary between the stick-slip and the permanent slip regime is drawn as a red curve. We also present as a light gray area the space where the block amplitude is larger than unity, i.e. when the block motion is amplified 
compared to the ground motion. This area is roughly centered around the undamped resonance line (dashed black line). The limit between the underdamped and the over-damped behavior is drawn as a vertical blue dashed line. By comparing the dimensionless forces (see Eqs. 5), we plot $\rho_{e f}=1$ (magenta), $\rho_{v f}=1$ (orange) and $\rho_{e v}=1$ (green). Thus, there exists an area where the friction force is dominant (light blue area), an area where the viscous dissipation force is dominant (light red area) and finally an area where the elastic restoring force is dominant (dashed area). It is interesting to remark that these three areas connect as a triple point (black circle) where the three forces are of equal magnitude. This occurs for an acceleration $\hat{\alpha}$ and a stiffness $\hat{k}=\eta \sqrt{\hat{\alpha} \mu_{s}}$.

As usual, the resonance is reached when then block amplitude reaches a maximum at a resonance acceleration $\alpha_{r}$. The blue curve represents the resonance line $\left(k_{r}, \alpha_{r}\right)$ where the block oscillation is maximum. For a large $k$ value, this line is asymptotic to $\alpha=k / \mu_{s}$, drawn as a dashed black line, the resonance condition without viscous dissipation.

Since this mapping depends on $\eta$, we present on Fig. 7 the behavior of the system for friction model 1 for different values of $\eta$. Above a weak value of $\eta$, the $\alpha_{S L}(k)$ curve becomes single-valued (see Fig. 7b for $\eta=0.05$ ). With an increase of $\eta$, the $\alpha_{S L}$ threshold increases, following the same trend as for the configuration 2 .

An increase of the viscous parameter implies obviously an extension of the viscous-dominated area (light red area), as shown on Fig. 7(e) for $\eta=1$. In this configuration, the triple point lies in the stick-slip domain and the $A>1$ area as well as the resonance line does not lie in the $(0<k<6,0<\alpha<6)$ 


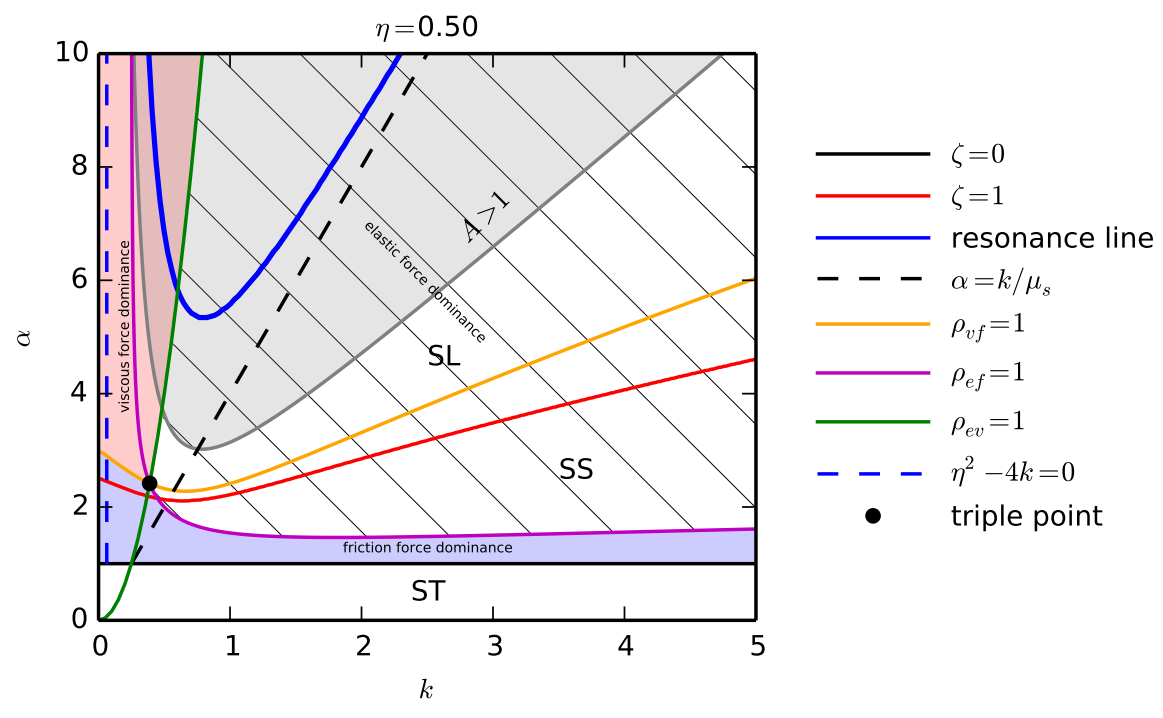

Figure 6: Configuration 4. Map of the block behavior in the $(k, \alpha)$ plane for $\eta=0.5$. Friction model 1. The light gray area sketches the condition where $A>1$. The friction force is dominant in the light blue area, the viscous dissipation force is dominant in the light red area, and the the hashed area represents the dominance of the elastic force. Also plotted is the triple point where the three forces are of equal magnitude. 
frame.

This mapping helps to characterize the block motion for any set of mechanical parameters $\left\{\alpha, k, \eta, \mu_{s}, \mu_{k}\right\}$. In particular, a good estimate of these parameters for an actual system may help to predict the occurence of a stickslip behavior or of a permanent slip behavior. Indeed, the stick-slip motion presents periodic singularities for the acceleration and thus produces strong force fluctuations. And this model also helps to predict the range of parameters for which the amplitude of the block is $A>1$, thus when the system amplifies the ground motion.

While the results presented in Figures 6 and 7 were computed for the friction model 1, the block amplitude (Fig. 8a, b) is weakly affected by the choice of the friction model, and for a weak dissipation $(\eta=0.01)$, the $A(\alpha)$ curve shows the same fluctuations as in configuration 3, above and below the resonant acceleration $\alpha_{r}$. The magnitude of these sub-harmonic and superharmonic resonances increases with the stiffness value (Fig. 8a for $k=1$ and Fig. $8 \mathrm{~b}$ for $k=2$ ). The influence of the viscous dissipation is clearly shown on Fig. 8c $(k=1)$ or Fig. $8 \mathrm{~d}(k=2)$, where the $\eta$ parameter is varied from 0.01 to 0.75 . When the dissipation increases, the $A(\alpha)$ fluctuations are damped, and for $\eta>0.1$ they even are undistinguishable.

\section{Conclusions}

We proposed an analytical and numerical study of the motion of a block linked by a spring, a dashpot and a friction contact to a rigid oscillating ground. For each configuration, we provided the solution of the motion equation, then analyzed these solutions by computing the slipping time ratio, 

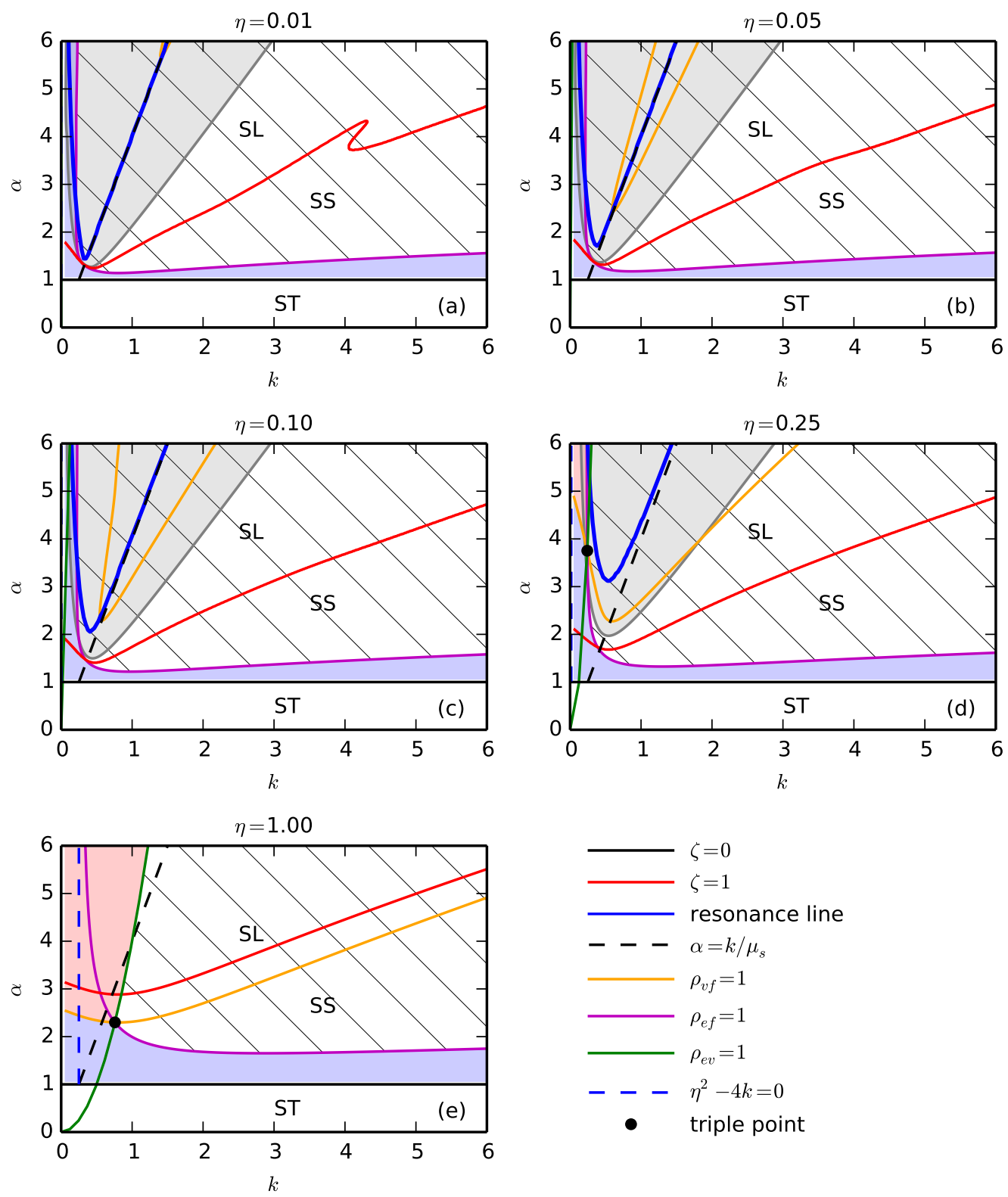

Figure 7: Configuration 4. Effect of $\eta$ on the motion regime in the $(k, \alpha)$ space for different values of $\eta$. For all plots, the friction model 1 is chosen. The light gray area sketches the condition where $A>1$. The friction force is dominant in the light blue area, the viscous dissipation force is dominant in the light red area, and the the hashed area represents the dominance of the elastic force. Also plotted is the triple point where the three forces are of equal magnitude. 

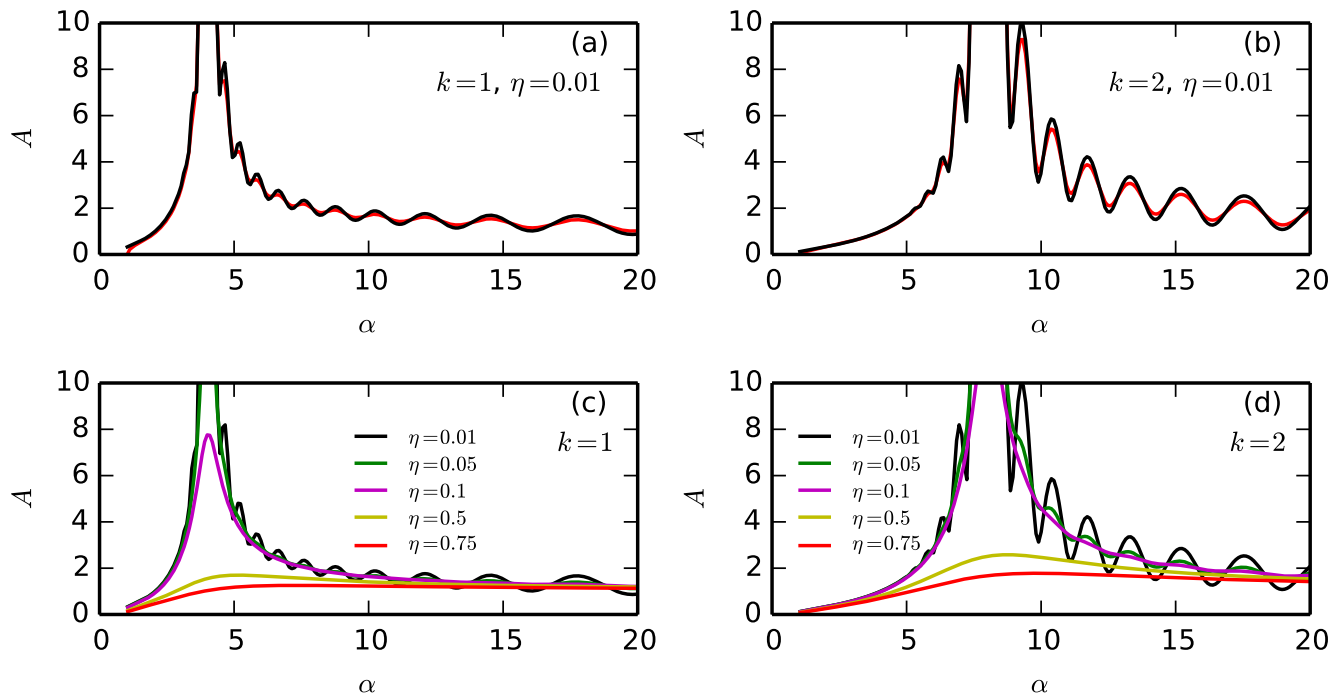

Figure 8: Configuration 4. The block amplitude vs $\alpha$ for the friction model 1 (red curves) and the friction model 2 (black curves) and for $k=1.0, \eta=0.01$ (a) and $k=2.0, \eta=0.01$ (b). Effect of $\eta$ for $k=1.0$ (c) and $k=2.0$ (d) for friction model 1. All curves correspond to an underdamped behavior. 
and comparing the energies and forces for a continuous range of parameters. From this work, the main conclusions are:

- the slipping limit has a non-trivial expression as soon as $k$ is non zero

- the friction model $\mu(\dot{x})$ has a weak effect on the behavior and on the block amplitude

- sub-harmonic and super-harmonic resonances due to the coupling of the friction and the stiffness

- for a given set of parameters $\left\{\alpha, k, \eta, \mu_{s}, \mu_{k}\right\}$, this model and its solving method provide a fast answer on the block behavior regarding the stickslip or permanent slipping regime, its amplitude and the dominant force in play.

With this comprehensive knowledge of the dominant energies and forces, and of the motion regimes (stick-slip or slipping regimes), this simple model of a single block may be extended to a vertical pile of identical or different blocks.

\section{Appendix: analytical solutions for configuration 4}

\subsection{Configuration 4a: underdamping}

When $\eta^{2}-4 k<0$, we introduce

$$
\omega_{k}=\frac{1}{2} \sqrt{4 k-\eta^{2}}, \quad D=\left(k-\omega^{2}\right)^{2}+\eta^{2} \omega^{2}
$$


so that the solution of the motion equation (2) writes

$$
\begin{aligned}
x(t)=\mathrm{e}^{-\frac{\eta}{2} t}\left[c_{1} \cos \left(\omega_{k} t\right)\right. & \left.+c_{2} \sin \left(\omega_{k} t\right)\right]-\frac{\mu_{k}}{k} \\
& -\frac{\omega^{2}}{D}\left[\left(k-\omega^{2}\right) \cos (\omega t+\varphi)+\eta \omega \sin (\omega t+\varphi)\right]
\end{aligned}
$$

where $c_{1}$ and $c_{2}$ are the solutions of

$$
\begin{aligned}
\mathrm{e}^{-\frac{\eta}{2} t_{0}} & \left(\begin{array}{cc}
a_{11} & a_{22} \\
a_{21} & a_{22}
\end{array}\right)\left(\begin{array}{c}
c_{1} \\
c_{2}
\end{array}\right) \\
= & \left(\begin{array}{c}
x_{0}+\frac{\mu_{k}}{k}+\frac{\omega^{2}}{D}\left[\left(k-\omega^{2}\right) \cos \left(\omega t_{0}+\varphi\right)+\eta \omega \sin \left(\omega t_{0}+\varphi\right)\right] \\
\dot{x}_{0}-\frac{\omega^{3}}{D}\left[\left(k-\omega^{2}\right) \sin \left(\omega t_{0}+\varphi\right)-\eta \omega \cos \left(\omega t_{0}+\varphi\right)\right]
\end{array}\right)
\end{aligned}
$$

with

$$
\begin{aligned}
& a_{11}=\omega_{k} \sin \left(\omega_{k} t_{0}\right)+\frac{\eta}{2} \cos \left(\omega_{k} t_{0}\right) \\
& a_{12}=\omega_{k} \cos \left(\omega_{k} t_{0}\right)-\frac{\eta}{2} \sin \left(\omega_{k} t_{0}\right) \\
& a_{21}=\cos \left(\omega_{k} t_{0}\right) \\
& a_{22}=\sin \left(\omega_{k} t_{0}\right)
\end{aligned}
$$

\subsection{Configuration 4b: critical damping}

When $\eta^{2}-4 k=0$, the solution of Eq. (2) is

$$
\begin{aligned}
x(t)=\left(c_{1}+\right. & \left.c_{2} t\right) \mathrm{e}^{-\sqrt{k} t}-\frac{\mu_{k}}{k} \\
& -\frac{\omega^{2}}{\left(k+\omega^{2}\right)^{2}}\left[\left(k-\omega^{2}\right) \cos (\omega t+\varphi)+2 \sqrt{k} \omega \sin (\omega t+\varphi)\right]
\end{aligned}
$$


when the block slips. The constants $c_{1}$ and $c_{2}$ are to be determined with the initial conditions.

$$
\begin{aligned}
& \mathrm{e}^{-\sqrt{k} t_{0}}\left(\begin{array}{cc}
1 & t_{0} \\
-\sqrt{k} & 1-\sqrt{k} t_{0}
\end{array}\right)\left(\begin{array}{c}
c_{1} \\
c_{2}
\end{array}\right) \\
& =\left(\begin{array}{c}
x_{0}+\frac{\mu_{k}}{k}+\frac{\omega^{2}}{\left(k+\omega^{2}\right)^{2}}\left[\left(k-\omega^{2}\right) \cos \left(\omega t_{0}+\varphi\right)+2 \sqrt{k} \omega \sin \left(\omega t_{0}+\varphi\right)\right] \\
\dot{x}_{0}+\frac{\omega^{3}}{\left(k+\omega^{2}\right)^{2}}\left[-\left(k-\omega^{2}\right) \sin \left(\omega t_{0}+\varphi\right)+2 \sqrt{k} \omega \cos \left(\omega t_{0}+\varphi\right)\right]
\end{array}\right)
\end{aligned}
$$

\subsection{Configuration 4c: overdamping}

The solution $x(t)$ for Eq. (2) can be analytically derived during the slipping motion for $\eta^{2}-4 k>0$ :

$$
x(t)=c_{1} \mathrm{e}^{-\frac{s_{1}}{2} t}+c_{2} \mathrm{e}^{-\frac{s_{2}}{2} t}-\frac{\mu_{k}}{k}-\frac{\omega^{2}}{D}\left[\left(k-\omega^{2}\right) \cos (\omega t+\varphi)+\eta \omega \sin (\omega t+\varphi)\right]
$$

with

$$
s_{1}=\eta+\sqrt{\eta^{2}-4 k}, \quad s_{2}=\eta-\sqrt{\eta^{2}-4 k} .
$$

The constants $c_{1}$ and $c_{2}$ are the solutions of

$$
\begin{gathered}
\left(\begin{array}{cc}
\mathrm{e}^{-\frac{s_{1}}{2} t_{0}} & \mathrm{e}^{-\frac{s_{2}}{2} t_{0}} \\
-\frac{1}{2} s_{1} \mathrm{e}^{-\frac{s_{1}}{2} t_{0}} & -\frac{1}{2} s_{2} \mathrm{e}^{-\frac{s_{2}}{2} t_{0}}
\end{array}\right)\left(\begin{array}{c}
c_{1} \\
c_{2}
\end{array}\right) \\
=\left(\begin{array}{c}
x_{0}+\frac{\mu_{k}}{k}+\frac{\omega^{2}}{D}\left[\left(k-\omega^{2}\right) \cos \left(\omega t_{0}+\varphi\right)+\eta \omega \sin \left(\omega t_{0}+\varphi\right)\right] \\
\dot{x}_{0}-\frac{\omega^{3}}{D}\left[\left(k-\omega^{2}\right) \sin \left(\omega t_{0}+\varphi\right)-\eta \omega \cos \left(\omega t_{0}+\varphi\right)\right]
\end{array}\right)
\end{gathered}
$$

\section{References}

[1] L. Papaloizou , P. Komodromos Planar investigation of the seismic response of ancient columns and colonnades with epistyles using a custom- 
made software. Soil Dynamics and earthquake engineering 29 (2009) $1437-1454$.

[2] G. Lancioni, S. Lenci, Q. Piattoni, E. Quagliarini. Dynamics and failure mechanisms of ancient masonry churches subjected to seismic actions by using the NSCD method: The case of the medieval church of S. Maria in Portuno. Engineering Structures 56 (2013) 1527-1546.

[3] K. Nakahara, T. Hisatoku, T. Nagase, Y. Takahashi. Earthquake response of ancient five-story pagoda structure of Horyu-Ji temple in Japan. 12WCEE (2000).

[4] C. Coladant. Seismic isolation of nuclear power plants - EDF's philosophy. Nuclear Engineering and Design 127 (1991) 243-251.

[5] M.B. Syed, L. Patisson, M. Curtido, B. Slee, S. Diaz. The challenging requirements of the ITER anti seismic bearings. Nuclear Engineering and Design 269 (2014) 212-216.

[6] L. Landi, G. Grazi, P.P. Diotallevi. Comparison of different models for friction pendulum isolators in structures subjected to horizontal and vertical ground motions. Soil Dynamics and Earthquake Engineering 81 (2016) 75-83.

[7] M.S. Hundal. Response of a base excited system with Coulomb and viscous friction. J. Sound and Vib. 64(3) 371-378 (1979).

[8] I. López, J.M. Busturia, H. Nijmeier. Energy dissipation of a friction damper. J. Sound and Vib. 278 (2004) 539561. 
[9] S. Chatterjee, Resonant locking in viscous and dry friction damper kinematically driving mechanical oscillators. J. Sound and Vib. 332 (2013) 34993516.

[10] N. Hinrichs, M. Oestreich and K. Popp, On the modelling of friction oscillators. J. Sound and Vib. 216(3) 435-459, 1998.

[11] J. P. Den Hartog, Forced vibrations with combined Coulomb and viscous friction, 1931 Transactions of the American Society of Mechanical Engineers 53 (1931), 107-115.

[12] D.W. Alspaugh. Analysis od Coulomb friction vibration dampers. J. Sound and Vib. 57(1) 65-78, 1978.

[13] B. Westermo, F. Udwadia. Periodic response of a sliding oscillator system to harmonic excitation. Earthquake Engineering and Structural Dynamics 11 (1983), 135-146.

[14] A. Benedetti, P. Sornay, B. Dalloz, M. Nicolas, An angular particle sliding down a transversally vibrated smooth plane, Physical Review E 85 (2012) 011307. 